\section{Райченко А.В.}

д-р экон. наук, профрессор, ФГБОУ ВО «Государственный университет управления», Москва

e-mail: a.v.raychenko@bk.ru

\section{Raychenko A.V.}

Doctor of Economics, Professor, FSBEI HE State University of Management, Moscow e-mail: a.v.raychenko@bk.ru

\section{Постановка методологии опережающего развития управленческого образования}

\begin{abstract}
Аннотация
Методологической основой научных исследований и профессионального управленческого образования служат классические и разрабатываемые подходы. Их постановка и использование осуществляются традиционно, инновационно и агрегировано, что становится конструктивным условием совершенствования процесса исследований и образования в сфере управления. С этих позиций осуществляется обоснование, постановка и применение методологии опережающего развития в процессно-ориентированном формате управления. Опережающая ориентация закладывает креативный потенциал мышления, формирует инновационную активность разработки, обеспечивает освоение и реализацию установки на достижение актуального результата. Приоритет опережающего развития обеспечивает освоение профессиональных знаний, практических умений и прикладных навыков обучающихся, конструктивными ресурсами разработки, постановки и реализации целей воздействия. Такая методология становится универсальной основой освоения и применения необходимых на практике инновационно ориентированных компетенций менеджера.
\end{abstract}

Ключевые слова:

методология, образование, опережение, развитие, управление.

\section{Statement of Managerial Education's Rapid Development Methodology}

\begin{abstract}
The methodological basis of scientific research and professional managerial education are the classical and elaborating approaches. Their statement and use are realized in traditional, innovative, and aggregated forms, and it becomes a constructive condition for improving the research and education process in the management area. From this positions justification, statement and application of a rapid development methodology in a process oriented management format are realized. Anticipatory orientation lays a creative potential of thinking, forms an innovative development activity, as well as provides an assimilation and implementation of objective for an urgent result achievement. The rapid development priority provides assimilation of professional knowledge, practical abilities and applied skills of learners with development's constructive resource, formulation and implementation of impact goals. This methodology becomes a universal basis for assimilation and application of necessary for the practice, innovatively oriented competences of a manager.
\end{abstract}

Keywords:

methodology, education, advance, development, management.
Общепринятой методологической основой научных исследований и профессионального управленческого образования служат как классические, исторически применяемые, так и вновь разрабатываемые научные подходы, теоретико-методологические концепции и поисково-исследовательские ориентации отечественных и зарубежных научных школ. Их определение, выбор, постановка и использование осуществляются как достаточно традиционно для освоения социально-экономических знаний, так и все чаще инновационно, например, по доминированию, приоритету, ориентации на постановку конкретной процедуры познания. При этом постановщики исследований, как правило, подчеркивают, что в любой из выстраиваемых парадигм могут свободно и конструктивно сочетаться не только классические или традиционные, но и иные, в том числе достаточно новые, методологические подходы, концепции, положения. Вариативность такого сочетания становится конструктивным условием совершенствования процесса исследований и образования в сфере управления, обеспечивающего ее поступательное развитие.

Постановка и применение методологии исследования, освоения и использования всего многообразия ресурсов управления традиционно определяется составом и сочетанием следующих классических подходов: исторический, системный, предметный, комплексный и т.д. Устоявшиеся конструкции такого сочетания давно и уверенно применяются в научных поисках и разработках большинства областей знаний, объекты и предметы которых достаточно статичны. При этом исследователи все чаще приходят к выводу, что с помощью статических 
подходов адекватно рассматриваются и осваиваются соответствующие им стабильные объекты, представляемые достигнутым ранее или прогнозируемым в будущем системным состоянием. К динамичным преобразованиям статичные подходы применяются с существенными оговорками, допусками, исключениями, обусловливающими, как правило, запаздывание в исследованиях и освоениях и соответствующую ему методологию «догоняющего» развития.

Между тем исторические исследования теории и практики конструктивных научных изысканий убедительно показывают, что постановка и применение методологии, изначально ориентированной на повторение пройденного, достижение достигнутого, продвижения «по накатанному», изначально обрекают на устойчивое положение «ведомого». При этом большинство негативных повторений оказывает деструктивное воздействие не только на методологию, но, прежде всего, на потенциал исследователей, их инновационную ориентацию и креативность мышления. Поэтому последнее время в науке и образовании традиционное стремление «догнать и перегнать» все шире и результативнее заменяет ориентацией на опережающее развитие.

Заимствование или мобилизация?

В современных условиях глобальной конкуренции эффективная профессиональная управленческая деятельность не может успешно строиться и результативно реализовываться только на основе бесконечного повторения, дублирования, адаптированного тиражирования традиционно применявшейся методологии. Бесперспективность попыток «догоняющего копирования» в глобальной конкурентной среде никогда не вызывала никаких сомнений у признанных ученых и практиков. Несмотря на это очевидное заключение, упрямое стремление к слепому повторению предлагаемого по форме и содержанию почему-то не формирует ожидаемого недоумения в современной профессиональной научнообразовательной среде. Напротив, активное продвижение не только уже критикуемых, но и изначально выхолощенных разработок принимается многими учеными и чиновниками за безусловное благо и конструктивную перспективу реального развития конкурентной способности отечественного подхода к управлению.

Развитие России в целом и отдельных отраслей ее социально-экономической организации в частности исторически не раз обращалось к общепризнанному передовому опыту зарубежных моделей технологии, экономики, права и других достижений [3]. Как правило, это обосновывалось признанием отставания отечественной науки практики конкретных отраслей и направлений развития от высших мировых достижений, стратегической неспособности собственной системы управления эффективно решать актуальные задачи функционирования и модернизации общества исключительно традиционными методами. Такой подход и сегодня общепризнанно объявляется не только допустимым, но совершенно необходимым, при условии открытого международного обмена доказавшими свои преимущества передовыми решениями. Вместе с тем к аналогичной оценке современной науки и практики управления мы приступили только в последнее время, и во многом в формате слепого заимствования далеко не всегда признанных, тем более проявивших свою эффективность, методологических подходов, форм, программ, разработок. Упрямо повторяя навязываемые подходы и решения, мы все чаще убеждаемся в очевидной или латентной заинтересованности их «промоутеров» не только в получаемых результатах, но и в прививаемых подходах [3].

В отличие от традиционно критической оценки отечественных реалий зарубежные разработчики таких подходов устойчиво продолжают живо интересоваться уже проявившими себя и только нарабатываемыми результатами постановки и применения эффективных достижений российской науки и практики управления. Это убедительно показывает, например, опыт международного сотрудничества PKК «Энергия», в ходе которого компетенции вчерашних выпускников ведущих отечественных вузов активно востребуются крупнейшими мировыми компаниями аэрокосмической отрасли [1]. Уже не первый год они привлекаются не только в качестве участников, но гораздо чаще и активнее в явной или завуалированной роли генераторов, модераторов, организаторов постановки и решения широкого спектра инновационных управленческих задач.

Такая не проходящая заинтересованность общепризнанных мировых научно-технических лидеров в компетентностном признании российских специалистов указывает на предпочтение ими реальных конкурентных преимуществ отечественного профессионального управления и методологии профильного образования. Например, госкорпорация «Ростехнология», объединяющая 663 организации, второе десятилетие успешно реализует конструктивную модель единого управления разработкой, производством и экспортом высокотехнологичной промышленной продукции посредством взаимодействия 8 холдинговых компаний в обороннопромышленном комплексе, 5 - в гражданских отраслях промышленности и 21 организации прямо- 
го управления. Это позволяет конструктивно планировать и обеспечивать стратегически ориентированную деятельность различных подразделений современными, высококвалифицированными менеджерами, подготовленными на общей управленческо-методологической основе к профессиональной деятельности в контексте опережающего развития [2].

Методологические подходы и методические решения соответствующей ориентации и значимости находят все более широкое применение в отечественном профессиональном образовании, что становится конструктивной методической основой освоения и закрепления инновационных профессиональных компетенций российского менеджмента. Сотрудничество Государственного университета управления (ГУУ) с этими и другими корпорациями показало, что применение обоснованного опережающим подходом инновационного формата интерактивной кооперации в программах научнопоискового и учебно-производственного взаимодействия позволило вместе с освоением профессиональных компетенций менеджера проводить отбор перспективных кандидатов на замещение вакантных должностей, взаимовыгодный в условиях активной коммерциализации подбора и подготовки персонала.

\section{Повторение или опережение?}

Каждому серьезному исследователю, а опытному практику тем более, понятно, что разрабатывать новое гораздо эффективнее на основе изучения и оценки, но вовсе не обязательно слепого копирования не только сомнительных, но даже самых передовых достижений науки и практики. Конструктивно оценивая стратегические преимущества, очевидно, что реально, главное успешно, конкурировать в современных рыночных условиях можно только на основе постановки и реализации подходов и разработок опережающего развития. Опираясь на мировые достижения, но не обязательно их слепо повторяя, перспективное опережение постановки и применения соответствующей методологии управленческого образования с нашей точки зрения должно обеспечиваться, прежде всего, приоритетами разработки и реализации эксклюзивных отечественных ключевых преимуществ. Именно они, как убедительно показывает история, формируют, развивают и обеспечивают в конечном счете реализацию конкурентно способных инновационных программ, не раз выводивших отечественные предприятия, организации и страну в целом из критически сложных, порой «безвыходных ситуаций.
На наш взгляд, сегодня важно как можно быстрее осознать, что в условиях современной глобальной конкуренции дело даже не в том, какого уровня методологические разработки мы пытаемся копировать. Принципиальной ошибкой может стать само предпочтение уже пройденного конкурентами уровня, потенциальной возможности опережающего развития на основе разработки и внедрения собственных, стратегически ориентированных инновационных решений. Подобный, исторически губительный, подход повсеместного заимствования активно продвигаемой стейкхолдерами «западной» методологии универсализации научного, производственного, образовательного процессов в области управления сегодня вряд ли способен обеспечить выявление, мобилизацию и освоение действительно перспективных ресурсов развития и соответствующих им конкурентных преимуществ отечественных ученых и практиков.

Научно обоснованное и исчерпывающее представление, адаптация и применение адекватной отечественным условиям, если не изначально самостоятельной методологии опережающего развития, особенно важны сегодня именно в управлении. Это наглядно подтверждается и тем, как вузы социально-экономической направленности ориентируют свои основные усилия на концептуальное обновление учебно-методического обеспечения управленческого образования, формирование базовых компетенций бакалавриантов, расширение спектра его конкретных прикладных профилей магистрантов, специализации и адаптации их к требованиям отечественных работодателей реального сектора экономики. Их состав и разнообразие обусловлены уже не только соответствующей диверсификацией объектов и субъектов воздействия, но предметной, функциональной, отраслевой, хронологической и другой специализацией содержания реальных процессов и систем управления [1].

Обоснование, постановка и, главное, применение методологии опережающего развития исключительно важны в целом, в едином процессно выстроенном формате, применительно к науке, образованию, профессиональной деятельности в сфере управления. Именно опережающая ориентация закладывает креативный потенциал мышления, формирует инновационную активность разработки, обеспечивает освоение и реализацию установки на достижение конечного результата. В условиях расширения реальной, а не «создаваемой для видимости» конкуренции формируемые опережающим подходом компетенции становятся ключевыми преимуществами 
научного и профессионального развития сегодня и в еще большей степени - завтра.

Проведенный анализ позволяет обоснованно сделать вывод о том, что только приоритет опережающего развития может обеспечить освоение таких, все активнее востребуемых сегодня, главное, ожидаемых завтра, профессиональных знаний, практических умений и прикладных навыков обучающихся конструктивными ресурсами разработки, постановки и реализации целей субъекта воздействия, стать универсальной основой освоения и применения необходимых практике, инновационно ориентированных компетенций менеджера.

\section{Тиражирование или модернизация?}

Функционально-приоритетный анализ исторической практики социально-экономического развития показывает, что стратегический успех национальных инноваций любого государства наряду с изучением и использованием мирового опыта всегда основывался преимущественно на мобилизации и применении эндемических ресурсов, составляющих эксклюзивное конкурентное преимущество конкретного общества [4]. В России к таким условиям сегодня общепризнанно относятся беспрецедентные территориальные и геологические ресурсы, устойчивые коллективно-корпоративные традиции, пробуждающийся предпринимательский потенциал, конструктивная социальная активность общества и многие другие, пока адекватно не оцененные отечественные конкурентные преимущества. Именно на них, их распознавании, мобилизации, постановке в статусе определяющих, на наш взгляд, должны основываться стратегически ориентированная эффективная методология опережающего развития научной и практической деятельности в сфере управления и конструктивно опирающееся на ее конкурентные преимущества категорийно-понятийное содержание профессионального образования.

В то же время рассматриваемая методология целенаправленно ориентирует на предметный учет, как всеобщих, так и эндемических деструктивных условий, оказывающих тормозящее воздействие на темпы развития опережающих трендов. К ним наряду с глобальными кризисными рефренами поступательного развития, наносящими отечественной экономике непропорционально существенные уроны, необходимо отнести и такие эндемические условия, как неустойчиво развивающиеся региональные ресурсы, латентные экономические взаимодействия, наконец, та же устойчиво пропагандируемая ориентация на безусловный приоритет всего импортного.
Так, понимание и постановка методологии современного научного представления и профессионального управленческого воздействия во многом определяются реальным соотношением и прикладным применением двух ключевых понятий: управление и менеджмент [3]. Пришедшее к нам в конце $\mathrm{XX}$ в. с Запада прикладное понимание менеджмента долгое время невольно или осознанно воспринималось и применялось отечественными исследователями и практиками как синоним управления. Это объяснялось введением в профессиональную коммуникацию, соответствующей теоретической проработке зарубежными учеными, западного понимания «стейкхолдерского» продвижения идей, которое дало «хлеб насущный» отечественным авторам «адаптированных, и не очень...» научно-образовательных публикаций.

Эта тенденция была активно подхвачена и «раскручена» традиционной для России «еще с пушкинских времен» модой на все зарубежное. Естественно, что применение понятия или просто наименования «менеджмент» быстро стало социальным «хитом», широко и разнообразно используемым на всех уровнях практически любых организаций, от «...эффективного менеджера - главы правительства», до «... менеджера влажной уборки помещений».

Между тем процессный подход отечественных исследователей и адаптируемое к реальным организационным отношениям прикладное применение термина «менеджмент» практиками, в конструктивном сочетании с использованием понятия «управление», постепенно расставляет все на свои места. Это позволяет сегодня уверенно определять, что менеджмент как процесс профессионального руководства подчиненными своей определяющей частью входит в содержание управления как целенаправленное воздействие руководителя организации на ее ресурсы. Непосредственно системное понимание менеджмента, как состава руководителей, еще более традиционно применяется учеными и практиками в официальном и в прикладном представлении субъекта управления организации. Очевидно, что в каждом из определений менеджмента, прежде всего, выделяются социальные основы, иерархическая природа его происхождения как процесса профессионального руководства организацией. Именно им во многом обусловливается развитие инновационного и креативного потенциала профессиональной деятельности руководителей отечественных организаций, формирование которого во многом определяется постановкой и применением методологии опережающего развития управленческого образования. 
Фундаментальная постановка и прагматичное приспособление к реальным российским условиям практики профессионального применения понятия «менеджмент» в исследовательском сообществе и различных организациях сходятся на единой для них социальной основе этого феномена. В одних случаях она традиционно определяет менеджмент как: «...управление через персонал, подчиненных, организации и т.п.», в других - предполагает постановку и применение понятия «профессиональное руководство» в качестве его наиболее точного синонима. В любом из этих подходов менеджментом, так или иначе, определяется широкая сфера формирования, рационализации и использования института, механизмов и ресурсов социально-психологических отношений руководства и персонала в управлении современной организацией.

\section{Управление или руководство?}

В реальной деятельности и в образовании методология опережающего развития все активнее востребуется и применяется в концептуальном обосновании, содержательном детерминировании и позиционировании все большего спектра социально-психологических и экономических целей, ресурсов, условий, факторов управления, подходов к оценке его эффективности и определению направлений совершенствования. Наиболее значимой с научной точки зрения задачей здесь является обоснование приоритета, становление концепции и обеспечение адаптации методологии опережающего развития исследований и освоения ресурсов управления как основы разработки и применения эффективных моделей профессионального руководства организацией [2].

Определяющее методологическое значение обучения такой деятельности отводится представлению, изучению и освоению перманентно совершенствующихся профессиональных компетенций менеджера как непосредственных опций прогнозирования, планирования, разработки, закрепления и применения знаний, умений и навыков обеспечения опережающего развития. В решении этих задач отечественному образованию предстоит более конструктивно ориентироваться не только на конкретные, но больше на перспективные прогнозы развития потребностей реальных работодателей, что обеспечивается соответствующим опережающим подходом. Именно эти задачи призваны решать профессиональные сообщества, все активнее включающиеся в формирование и формулирование состава и содержания, осваиваемых обучающимися компетенций [1].
Управление в целом и менеджмент, как его ключевая составляющая, в частности, процедурно определяют современные бизнес-процессы как целенаправленные последовательности конструктивных преобразований. Практика постановки и применения ресурсов методологии опережающего развития убедительно показала, что в их исследовании и освоении эффективнее использовать следующие подходы: процессный, сканирующий, селективный и т.п. Их востребование, разработка и применение обусловлены динамичной природой возникновения, процессной формой представления и турбулентными условиями реализации целенаправленного воздействия [5]. Они приоритетно ориентированы на инновационную постановку и прорывное решение фундаментальных задач, обеспечивают, как правило, наиболее оригинальное и контрастное представление содержания каждой отдельной области знаний.

В постановке методологии опережающего развития необходимо понимать, что ее эффективность определяется не столько составом или структурой, но, прежде всего, приоритетом выбора и применения того или иного подхода в статусе ключевого, как стратегически ориентированной, процессуальной основы организации исследований и освоений области менеджмента. С позиций анализа и оценки каждого из выбираемых в той или иной степени актуально востребованных подходов важно подчеркнуть необходимость четкого обоснования того, что ключевым для конкретной методологии становится не их состав. Определяющую роль в ней играет конструктивная конфигурация сочетания системного и процессного подходов исследованиям, задающая приоритеты использования агрегируемых ими ресурсов в ходе научного поиска, учебного освоения и прикладного применения получаемых результатов.

\section{Система или процесс?}

Постановке и разрешению этого философского вопроса посвящены фундаментальные и прикладные исследования и обоснования в разных областях науки и сферах практической деятельности.

Их принципиальное осмысление приводит к признанию очевидности логики следующих положений:

1. Любая система является результатом процесса ее создания.

2. Далеко не каждый процесс заканчивается созданием системы.

3. Значит, N-1 процесс первичен, при N - первой созданной системе. 
Очевидно, что это заключение особенно важно в постановке и применении исследований и освоении всего разнообразия проявлений управления как процесса целенаправленного воздействия. Распространение системного восприятия управления объясняется более простым пониманием статичных представлений, отражающих в большинстве представлений уже прошедшие, а в остальных - возможные в будущем, уже поэтому не соответствующие настоящему состоянию.

Принципиальным отличием концептуальных основ постановки методологии опережающего развития управленческого образования, на наш взгляд, должна стать замена приоритета системного подхода, процессной альтернативой, обусловленной соответствующим пониманием, представлением и применением управления как процесса целенаправленного воздействия. Этим обусловливается необходимость разработки научной концепции приоритета процессного подхода в целом и широкого спектра его модификаций в частности, объединенных обоснованием выбираемой методологии и отражающих универсальные возможности механизма его постановки, представления, адаптации и применения. Очевидно, прежде всего, приоритет процессного подхода должен обеспечивать именно универсальность постановки и адекватность применения разрабатываемой и применяемой процедурной, проектной или иной конфигурации к исследованию и освоению выделяемых реалий и прогнозируемых перспектив опережающего развития.

Приоритет процессных построений исследований наиболее адекватен управленческому представлению, выражающемуся в целях, направлениях, последовательностях разработки и реализации воздействия. В его постановке и применении контрастно проявляются опускаемые многими учеными и практиками существенные отличия процессного формата постановки и осуществления управления в целом и менеджмента в частности от системного. Они выявляются в процессном представлении управления как целенаправленного воздействия в формате конструирования процедуры, в отличие от системного, раскрывающего, как правило, перманентно устаревающее представление в формате прошедшего состояния структуры. На этом основании все чаще именно процессный формат востребован в исследованиях и разработках перспективно нацеленного поиска, компетентностно ориентированной конфигурации, стратегически разрабатываемого и предметно направленного воздействия менеджмента, что предъявляет соответствующие требования к определению содержания принципов методологии опережающего развития.

Рассматривая их вновь, необходимо вернуться к оценке принципиальных различий в постановке и применении системного и процессного подходов, в качестве ключевых приоритетов построения методологии исследований и освоений ресурсов менеджмента. В ней следует четко выделить, что в сравнении приведенных выше представлений проявляется различие в том, что в разрезе процессного подхода приоритетно выделяется процедура как последовательность действий по достижению цели. Результатом большинства системных исследований и разработок становятся структуры, классификации, формы и т.п. Этот вывод необходимо проецировать на понимание того, что в реальных условиях менеджмент наиболее привычно воспринимается именно процедурой разработки, принятия и реализации решения, обеспечивающего достижение поставленной цели.

В ходе постановки и применения методологии опережающего развития исследований и освоений ресурсов менеджмента и управления необходимо постоянно учитывать выделенные различия в форматах и результатах представления и оценки процессного и системного подходов [3]. Так, с позиций процессного подхода принимаемое решение представляется, прежде всего, интеллектуальной моделью процедурной организации действий, в отличие от того, как оно становится основополагающей формой проявления результатов воздействия в виде системы в целом или ее составляющих в частности. Очевидно, что этим обусловливается приоритет разработки и реализации процессных подходов, форматов, проекций, построений и реализаций, обеспечивающих последовательную, временную, технологическую и другие процедурные ориентации разработки и реализации воздействий.

\section{Сочетание или приоритет?}

В реальном управлении жизненный цикл решения как теоретическое обоснование процедуры подготовки, выработки и принятия варианта воздействия представляет собой модель процесса, определенным образом формирующейся последовательности разработки и осуществления воздействия. При этом неважно, относится ли данное решение к целенаправленному воздействию на внешнюю, относительно объекта, систему или представляет собой новый вариант внутренней организации; оно в любом случае является интеллектуально формируемой моделью представления процесса целенаправленного воздействия. Это заключение выделя- 
ет наиболее конструктивные методологические возможности постановки и применения приоритета процессного подхода к исследованиям и освоениям ресурсов современного управления.

Приоритет процессного подхода обусловливает, формирует, реализует и модернизирует структурное представление систем на основе оптимизации, закрепления и сопровождения выполнения, обеспечиваемых, поддерживаемых, осуществляющихся ими процессов [3]. На этой основе инновационные процессные исследования, долгое время носившие эмпирический характер, все чаще становятся исходным условием прогнозирования и проектирования приоритетных преобразований и перспективного развития организации, организационных изменений и управления ими. Более того, любая процедура, одним из своих наиболее адаптированных, прагматических определений, непосредственно квалифицируется как обусловленное во времени и кооперации сочетание различных форм взаимодействия. Очевидно, что исследование и освоение таких взаимодействий по определению приоритетно востребуют постановку и применение процессного подхода, обеспечивающего адекватный формат их выявления, анализа, представления.

Исследование палитры основных проявлений агрегаций и коопераций разнообразных составляющих процессного подхода позволяет представить все его ресурсное многообразие и в конечном виде позиционировать его в единой логически выверенной модели исследования, обучения, воздействия. Такое обоснование, представление и позиционирование становится этапом, непосредственно раскрывающим адаптацию постановки и применения процессного подхода к исследованию разнообразия, разработке модификаций, совершенствованию и развитию связей между составляющими целенаправленного воздействия. Процессно-ориентированными подходами к постановке и применению методологии управления стали наиболее востребованные процедурами познания, образования и профессиональной деятельности в сфере управления устойчивые конструкции сочетания, кооперации, агрегирования правил, норм, рекомендаций, к которым, прежде всего, можно отнести представленные в табл. 1 следующие модификации.

Помимо модификаций, представленных в табл. 1, исключительно широкий спектр допустимого разнообразия формируемых модификаций постановки и применения процессного подхода методологии опережающего развития управления основывается на ключевых процедурных свойствах построения целенаправленного воздействия [3]. В актуальных научных исследованиях он достаточно квалифицированно представлен перечислением палитры: последовательное, параллельное, цикличное, реверсивное, ротационное, сканирующее, декомпозированное, детерминированное, дифференцированное, интеграционное или иное специализированное построение процессного взаимодействия. Эти и развивающие их применение модификации представляют собой содержание и разнообразие построения динамических моделей базовых процедур исследования управления. Возможные формы организации такого взаимодействия отражаются не столько рядом вариаций, приведенных в табл. 1, сколько раскрывающими их сочетания проявлениями, которые могут формироваться в целенаправленном процессе их адаптации и преобразования применительно к решаемым задачам.

Позиционируя, сочетая и реализуя приведенные в табл. 1 вариации процессного подхода, ученым научно-исследовательского института управления ГУУ удалось модернизировать сложившийся и широко применяемый спектр образовательных программ, методик, конструкций на основе применения инновацио нно ориентированной методологии в условиях постоянно развивающихся исследовательского, учебного и административного процессов управления. Это позволило наиболее полно и достоверно выявить, исследовать, обосновать, разработать, позиционировать и предоставить для последующего применения достаточно широкий спектр как классических и традиционных, так и инновационных подходов к исследованиям и освоениям ресурсов менеджмента [3].

Примером успешной реализации таких разработок является практика востребованных на протяжении двадцати лет программ проведения деловых игр в системе ресурса, резерва и состава руководящих кадров ОАО РКК «Энергия» [2]. Основанные на процессном подходе построения исследований, представлений, функционирования и модернизации процедуры проведения деловых игр стали универсальным форматом обучения, оценки и стимулирования наиболее перспективных кандидатов на замещение вакантных должностей руководителей подразделений корпорации среднего и нижнего уровней управления. Вместе с тем это позволило наиболее полно и достоверно выявлять, исследовать, позиционировать и предоставлять для распространения и адаптированного применения исключительно широкий спектр не только классических и традиционных, но в большей степени оригинальных и инновационных подходов и решений. Они эффективно способствуют выявлению, освоению и 
Модификации процессного подхода постановки и применения методологии опережающего развития управленческого образования

\begin{tabular}{|l|l|}
\hline \multicolumn{1}{|c|}{ Модификация } & \multicolumn{1}{|c|}{ Сущность и содержание модификации процессного подхода } \\
\hline Ретроспективная & $\begin{array}{l}\text { Хронологически выстроенное представление последовательности накопления, анализа и оценки применения } \\
\text { исследований и результатов }\end{array}$ \\
\hline Прогнозная & $\begin{array}{l}\text { Выявление, обоснование и построение ключевых тенденций развития, установление перспективных } \\
\text { направлений модернизации }\end{array}$ \\
\hline Аналитическая & $\begin{array}{l}\text { Исследование, выявление, идентификация, представление процедур построения состава и содержания } \\
\text { целенаправленного воздействия }\end{array}$ \\
\hline Трендовая & $\begin{array}{l}\text { Определение направления, скорости, сущности и содержания происходящих и ожидаемых изменений и } \\
\text { конвергенций }\end{array}$ \\
\hline Приоритетная & $\begin{array}{l}\text { Исследование, прогнозирование, выделение, позиционирование и ориентация обоснований и построений на } \\
\text { первостепенные требования }\end{array}$ \\
\hline Проектная & $\begin{array}{l}\text { Целеполагание, декомпозиция, верификация, этапирование содержания деятельности с координацией и } \\
\text { адаптацией исследований }\end{array}$ \\
\hline Процедурная & $\begin{array}{l}\text { Разработка и построение последовательности этапов осуществления и условий перехода построением } \\
\text { динамичной модели управления }\end{array}$ \\
\hline Операционная & $\begin{array}{l}\text { Декомпозиция, специализация, детализация, исследований и действий с целью последующей кооперации } \\
\text { в ходе достижения результата }\end{array}$ \\
\hline Оптимизационная & $\begin{array}{l}\text { Определение, оценка, сопоставление, рационализация повышение результатов, качества, оперативности, } \\
\text { эффрективности }\end{array}$ \\
\hline Маршрутная & $\begin{array}{l}\text { Разработка, расчет, согласование, построение дорожной карты разработки, принятия и реализации программы } \\
\text { деятельности }\end{array}$ \\
\hline Модельная & $\begin{array}{l}\text { Обоснование, сопоставление, разработка, построение и применение механизма конфригурации } \\
\text { функционирования организации }\end{array}$ \\
\hline Коммуникационная & $\begin{array}{l}\text { Интеграция позиционирования, функционирования и взаимодействия механизмов, программ и кооперации } \\
\text { образовательных ресурсов }\end{array}$ \\
\hline
\end{tabular}

применению современных ресурсов управления в сферах научных разработок, образовательных процессов и функционирования ряда производственных предприятий реального сектора экономики [2].

Наиболее перспективные и востребованные сегодня разработки теории и практики управления представляют собой не только адаптированные к принципам процессной ориентации, но и перманентно модернизируемые ресурсы постановки и применения методологии опережающего развития. Востребование, адаптация и использование ее возможностей опираются на результаты фундаментальных и прикладных исследований соответствующих разделов ряда бурно развивающихся самостоятельных наук и учебных дисциплин [3]. В настоящей публикации мы только определяем и обосновываем процессный подход в качестве важнейшей конструктивной основы методологии опережающего развития управления, адресуя читателей, заинтересованных в более подробном ознакомлении с подобными разработками в других областях знаний, к соответствующим научным источникам [1].

\section{Цель или средство?}

Актуальная методология представляет собой не только адаптированный к принципам процессной ориентации, но и перманентно модернизируемый интеллектуальный ресурс постановки и применения подходов, методов, средств опережающего развития исследований, освоений и действий в сфере управления. Этим обусловливается изначальное востребование, приоритетное позиционирование и стратегическая ориентация методологии опережающего развития в качестве основного исследовательского ресурса выявления, освоения и применения возможностей целенаправленного воздействия. Исходя из этого обоснование и совершенствование методологии становится одной из фундаментальных целей, а ее освоение и применение - конструктивным средством осуществления научных исследований, обучающих освоений и профессиональных применений теории и практики целенаправленного воздействия.

Приоритеты постановки и применения процессного подхода, процедурного позиционирования, сопровождающего регулирования и соответствующих ресурсов в сфере управления обусловливаются востребованием актуальных фундаментальных и прикладных исследований и разрешений динамично развивающихся противоречий социально-экономических процессов и систем. Этими противоречиями определяется необходимость перманентной модернизации состава и содержания ресурсов ме- 
тодологии опережающего развития, обеспечивающая актуальность ее востребования и применения не только в образовании или прикладной деятельности в сфере управления, но, прежде всего, в ориентированных на разработку инновационных подходов и принципиальных решений опережающих фундаментальных научных исследованиий.

Проводимые в научно-исследовательском институте управления ГУУ фундаментальные исследования и прикладные разработки постановки и применения процессного подхода в целом и процедурного формата во всем его разнообразии, в частности, позволяют оснастить методологию опережающего развития конструктивно новым инструментарием. На наш взгляд, именно он способен обеспечить исчерпывающий спектр вариантов агрегирования, кооперации, сочетания путей и конкретных способов целенаправленного воздействия, логически обусловливаемого упорядочиванием последовательности выработки, принятия и реализации решения как содержания этого воздействия на объект исследования и управления. Существенно обогащая и развивая теорию и практику постановки и применения процессного подхода в исследовании, образовании и целенаправленном воздействии в сфере управления, процессный подход раскрывает новые возможности разработки и адаптации методологии опережающего развития в целом.

Изучение практики разработки и реализации целенаправленного воздействия убедительно показывает, что неуклонно возрастающая актуальность постановки и применения процессного подхода в формате методологии опережающего развития исследований, образования и профессиональной деятельности в сфере управления основывается на изначально процедурном представлении и последовательно обусловленном построении выработки, принятия и реализации управленческих решений. Как в традиционных, так и в инновационных научных исследованиях они обосновываются и применяются широким спектром форматов процессных построений, представляющих всю палитру возможных преобразований, от генезиса до онтогенеза. Очевидно, что непреходящая востребованность процессных представлений и применений обусловливает соответствующее позиционирование и востребование основывающихся на них подходов, методов, инструментов в сфере актуальных профессиональных управленческих исследований и воздействий.

Проведенный анализ постановки и применения методологии управления определяет ключевое место и ведущую роль выбираемых и применяемых в качестве приоритетного подхода, конструкции сочетания, доминанты комплекса подходов постановки и применения всего методологического обеспечения опережающего развития. Это обусловливает особую ответственность каждого конкретного исследователя и практики управления за выбор и применение состава, содержания и агрегации ключевых и обеспечивающих подходов, необходимость обоснования его авторского отношения к планированию и осуществлению научных разработок, их нацеленность на конкретный результат. В образовании это обусловливает и ряд основополагающих требований обеспечения всестороннего и взвешенного отношения к выбору и применению непосредственного состава и реальной конфигурации сочетания научных подходов в контексте все активнее востребуемой профессиональной средой методологии опережающего развития.

\section{Литература}

1. Afanasiev V.Ya. Interactive development of managerial competencies [Text]: Monographic / V.Y. Afanasiev [et al.]. Rome: Eurilink, 2015.

2. Афанасьев В.Я. Инженерно-экономическая ориентация управленческого образования [Текст] / В.Я. Афанасьев, А.В. Райченко // Вестник университета. - 2015. - № 1.

3. Афанасьев В.Я. Методология фундаментальных и прикладных исследований управления [Текст]: монография / В.Я. Афанасьев, А.В. Райченко. - М.: ГУУ, 2014.

4. Райченко А.В. Генезис менеджмента [Текст]: монография / А.В. Райченко. - М.: ГУУ, 2015.

5. Райченко А.В. Концепция единого теоретико-методологического комплекса исследований, образования и деятельности в сфере управления [Текст] / А.В. Райченко. - В кн.: Актуальные проблемы управления-2015. - М.: ГУУ. Вып. 1 сборника международной научно-практической конференции, 2015.

\section{References}

1. Afanasiev V.Y., Mitrofanova E.A., Raychenko A.V. Interactive development of managerial competencies. Rome, Eurilink Publ., 2015.

2. Afanas'ev V.Ya., Raychenko A.V. Engineering and economic orientation of management education. Vestnik universiteta [University Bulletin]. 2015, V. 1. (in Russian)

3. Afanas'ev V.Ya., Raychenko A.V. Metodologiya fundamental'nykh i prikladnykh issledovaniy upravleniya [The methodology of basic and applied research management]. Moscow, 2014.

4. Raychenko A.V. Genezis menedzhmenta [Genesis management]. Moscow, 2015.

5. Raychenko A.V. The concept of a unified theoretical and methodological complex of research, education and activities in the field of management. Aktual'nye problemy upravleniya-2015 [Actual problems of management-2015]. Moscow, 2015, V. 1. (in Russian) 